\title{
Bone Health in HIV-Infected Children, Adolescents and Young Adults: A Systematic Review
}

Stephen M Arpadi ${ }^{1,2,3^{*}}$, Stephanie Shiau ${ }^{1,2}$, Charlotte Marx-Arpadi ${ }^{1}$ and Michael T Yin ${ }^{4}$

1 Gertrude H. Sergievsky Center, Columbia University Medical Center, New York, NY, USA

2Department of Epidemiology, Mailman School of Public Health, Columbia University Medical Center, New York, NY, USA

3Department of Pediatrics, Columbia University Medical Center, New York, NY, USA

4Department of Medicine, Division of Infectious Disease, Columbia University Medical Center, New York, NY, USA

*Corresponding author: Stephen M. Arpadi, MD, MS, Gertrude H. Sergievsky Center, Columbia University, 630 W. 168th Street, PH 19-114, New York, NY 10032, USA, Tel: 2123052384; Fax: 2123052526; E-mail: sma2@columbia.edu

Received date: August 22, 2014; Accepted date: October 30, 2014; Published date: November 09, 2014

Copyright: (C) 2014 Arpadi SM, et al. This is an open-access article distributed under the terms of the Creative Commons Attribution License, which permits unrestricted use, distribution, and reproduction in any medium, provided the original author and source are credited.

\begin{abstract}
Background: Children and adolescent, who acquire HIV infection early in life either perinatally, from contaminated blood products or via sexual transmission, have the greatest cumulative exposure to the negative direct and indirect effects of HIV infection and ART on bone. This may lead to increased lifetime risk for osteoporosis and fracture. We conducted a systematic review to evaluate the literature on bone health in children and adolescents with HIV.
\end{abstract}

Methods: We performed a comprehensive search of the Medline, Scopus, and Cochrane Library databases (up to April 1, 2014) for studies that reported on bone imaging or bone fractures in HIV-infected children, adolescents, or young adults.

Results: A total of 32 publications met our inclusion criteria. Seventeen studies were cross-sectional and 15 were longitudinal. The majority of studies were conducted in high-income countries, three in middle-income countries and none in low-income countries. Overall, the studies we reviewed indicate that measures of bone mass are reduced, with increased prevalence of low BMD in children and adolescents with HIV. However, the studies are highly variable with respect to comparison sources, measurement methods, adjustment techniques for body size or growth retardation, and highlighted risk factors, including aspects related to medication exposures as well as the effects of HIV infection per se.

Conclusions: HIV infection appears to be associated with decreased bone accrual throughout childhood and adolescence. Sub-optimal bone accrual may persist through childhood and adolescence and result in reduced peak bone mass, an important determinant of future risk of osteoporosis and fracture. Important areas for future research include evaluation of bone mass, bone quality and fracture risk across the life course among those with early-life infection with HIV, particularly in resource-limited settings where the majority of children with HIV live.

Keywords: HIV; Perinatal; Children; Osteoporosis; Bone

\section{Introduction}

Low bone mineral density (BMD) and increased fracture rates have been reported in HIV-infected individuals, particularly in older men and postmenopausal women [1-5]. Decreased bone mass has also been reported in children, adolescents and young adults who acquire HIV early in life from perinatal or sexual transmission [6,7]. Multiple factors appear to be involved, including effects of HIV-1 viral proteins, inflammatory cytokines and antiretroviral therapy (ART) on bone cells and bone turnover [8-14]. Children and adolescents infected with HIV have the greatest cumulative exposure to the negative direct and indirect effects of HIV infection and ART on bone metabolism, which may lead to an increase in the lifetime risk for osteoporosis and fracture.

As approximately $85-90 \%$ of final adult bone mass is attained during childhood and adolescence, impaired bone accrual during these critical periods of skeletal maturation may compromise peak bone mass (PBM), the maximum amount of bony tissue at the end of skeletal maturation, which is an important determinant of adult osteoporosis and fracture risk [15-17]. Potent ART has transformed HIV from a fatal illness to a manageable chronic infection with near normal life expectancy $[18,19]$. With approximately 6 million people age 24 and under living with HIV and possibly more than 1.5 million on ART [20], the long-term outcome of bone development across the life course and causes of suboptimal bone development have emerged as an important areas of investigation. Thus, the aim of this study was to systematically review the literature, summarize the publications concerning bone health in children, adolescents, and young adults with HIV, and identify areas where further research is needed. 


\section{Methods and Materials}

\section{Search process}

We conducted a comprehensive systematic search for original publications reporting bone imaging or bone fractures in HIV-infected children, adolescents, or young adults. Journal articles were retrieved using Medline, Scopus and The Cochrane Library databases up to April 1, 2014. Studies that were electronically published ahead of print publication during this time period were eligible for inclusion. Specific search criteria are reported in Table 1.

\begin{tabular}{|c|c|c|c|}
\hline Database & Time Period & Search Terms & \# \\
\hline Medline & Up to April 1, 2014 & \begin{tabular}{lrr} 
("bone & and & \multicolumn{2}{c}{ bones"[MeSH } \\
Terms] OR & ("bone"[All & Fields] \\
AND & "bones"[All Fields]) & OR \\
"bone and bones"[All & Fields] \\
OR "bone"[All & Fields]) & AND \\
("hiv"[MeSH & Terms] & OR \\
"hiv"[All & Fields]) & AND \\
(("child"[MeSH & Terms] & OR \\
"child"[All & Fields] & OR \\
"children"[All & Fields]) & OR \\
("adolescent"[MeSH Terms] OR \\
"adolescent"[All & Fields] & OR \\
"adolescents"[All & Fields]) & OR \\
("young adult"[MeSH & Terms] \\
OR ("young"[All & Fields] & AND \\
"adult"[All Fields]) OR & "young \\
adult"[All & Fields] & OR \\
("young"[All & Fields] & AND \\
"adults"[All Fields]) OR & "young \\
adults"[All Fields])) &
\end{tabular} & 676 \\
\hline Scopus & Up to April 1, 2014 & $\begin{array}{l}\text { HIV AND bone AND (children } \\
\text { OR adolescents OR young } \\
\text { adults) in "Article Title, Abstract } \\
\text { and Keywords" }\end{array}$ & 668 \\
\hline $\begin{array}{l}\text { Cochrane } \\
\text { Library }\end{array}$ & Up to April 1, 2014 & HIV and bone & 6 \\
\hline
\end{tabular}

Table 1: Database search terms

\section{Study selection and data collection}

Two investigators (SS, CMA) independently reviewed all publications for inclusion. A study was eligible for inclusion if it reported on bone imaging or bone fractures in HIV-infected children, adolescents, or young adults (up to age 24) and had a full-text article in English. Studies with and without a control group were included, however, studies were excluded if they had less than five HIV-infected individuals.

The following data was abstracted, when available, from each publication that met the inclusion criteria: authors, year of publication, study location and design, total number of participants, number of HIV-infected participants and controls or reference source used for comparison (if applicable). Detailed information on HIV-infected participants, including age, sex, stage of biologic maturation, race/ ethnicity, and transmission status were obtained. Where available, we recorded information on bone fractures as well as imaging methods used including dual-energy X-ray absorptiometry (DXA), quantitative computed tomography (QCT), or quantitative ultrasound (QUS). We also recorded specific measurements, including bone mineral content $(\mathrm{BMC})$, bone mineral density (BMD), bone area (BA), cortical and trabecular thickness, speed of sound (SoS), broadband ultrasound attenuation (BUA), bone transmission time (BTT), and skeletal sites, including whole body (WB), lumbar spine (LS), femoral neck (FN), total hip (TH), 1/3 distal radius (R13), tibia, phalanges, and calcaneus. In addition, we recorded techniques for adjustments of bone measures, risk factors, and key findings.

\section{Results}

\section{Selection of studies and study characteristics}

Results from the literature search and study selection process are shown in Figure 1. A total of 32 articles met inclusion criteria. The characteristics of these studies, published between 2001 and 2014, are summarized in Table 2. Most studies were conducted in high income countries, including the United States [6,7,21-32], Italy [33-44], Canada [45], and the Netherlands [46]. Three studies were reported from middle-income countries, including one from Thailand [47] and two from Brazil $[48,49]$. One study was conducted in three countries: the United States, Brazil, and Panama [50]. No studies were conducted in low-income countries. While the ethnic and racial breakdown varied across studies, several included only white/Caucasian participants [33,35-40,43]. The source of comparison group varied across studies. Many recruited a healthy control group [31,35,37-40] and several included age, sex, ethnicity, or pubertal stage as criteria for control group selection [6,7,25,36,44,51]. Two studies enrolled children known to be HIV-uninfected but exposed to HIV in utero as a control group $[29,30]$ and one study recruited HIV-uninfected siblings [24]. Other studies drew comparisons with a number of existing cross-sectional or longitudinal normative databases comprised of healthy children from single or multiple study sites $[21-23,26-28,34,45,47]$ or provided by the densitometer manufacturer $[24,41,43,46,48,49]$. Bone densitometry by DXA was the predominant method for evaluation. A sole manufacturer densitometer (Hologic, Bedford, MA or GE Lunar, Madison, WI) was used in all but 7 studies $[6,7,24,30,47,50]$ which used both [52].

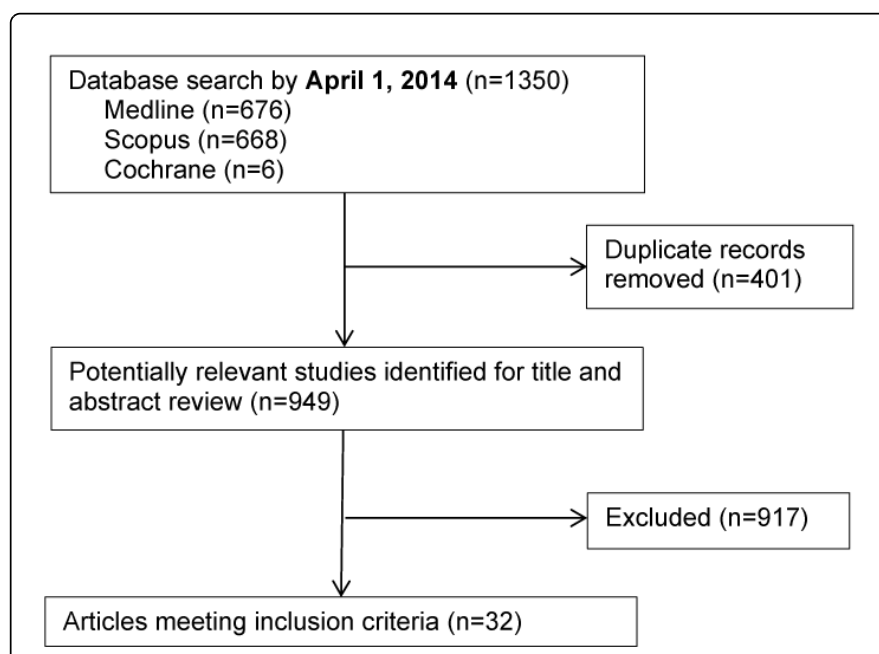

Figure 1: Flow diagram of literature search and study selection

A total of 17 cross-sectional and 15 longitudinal studies were identified (Table 2). Among cross-sectional studies, 16 reported significant decreases in one or more bone measures in those with HIV including lower BMC and BMD, both WB as well as LS. Some, but not 
all, reported bone outcomes adjusted for variables such as age, sex, race/ethnicity, weight, height, body composition, and pubertal status. A recent study by DiMeglio et al. observed that Z-score differences between those with HIV and the comparison group were attenuated after adjusting for sex, race/ethnicity, weight, height, and puberty stage [30], which was similar to an earlier study which adjusted for sex, weight, and bone area [34]. Jacobson et al. found that reductions in bone mass were most marked among boys who achieved biological maturation [6]. Sex differences, however, were not confirmed in a recent study conducted in Brazil [48].

A longitudinal study of $32 \mathrm{HIV}$-infected children aged 6.3 to 17.7 years on long-term ART observed that although WB BMD increased over time, the annual increment was less in those with HIV compared to healthy controls [35]. A small prospective study of 18 perinatally HIV-infected children (mean age 11.3 years) the majority of whom were on ART ( $>80 \%)$, found that while all healthy control subjects had increased or stable WB BMD over a 1-3 year period, this was true for only $44 \%$ of the HIV-infected group ( $\mathrm{p}=0.09$ ) [24]. A larger study with 66 HIV-infected subjects, median age 6.7 years, with DXA assessments at 2-3 year intervals reported improved LS and FN BMD Z-scores during follow up [46]. A study of older perinatally-infected children ages 11-16 years (mean age 13.6 years) at baseline with 1 and 2 year
DXA assessments found a similar result with BMD [45]. Declines in BMD have also been reported in several studies and appear to be primarily associated with changes in ART $[23,26,50]$.

Although osteoporosis, i.e. low bone mass and fragility fractures [53], is not reported in children and adolescents with HIV, increased prevalence of low BMD (Z-score $\leq-2.0)$ alone was reported in 6 studies from high and middle-income countries including Italy [30], The Netherlands [46], the United States [25], Brazil [48, 49], and Thailand [47]. In a study of $101 \mathrm{HIV}$-infected Thai adolescents ages 12-20 years, $24 \%$ met criteria for low WB BMD [47]. Studies conducted in Brazil reported low WB and or LS BMD in $32 \%$ of 74 perinatally HIVinfected children (mean age 17.3 years) [49] and low WB BMD in $17 \%$ of 48 children (mean age 12.7 years) [48]. Much lower prevalence was observed by DiMeglio et al. who found in a study of 350 Italian children (mean age 12.6 years) that $7 \%$ had low WB BMD and $4 \%$ had low LS BMD compared to $1 \%$ for both WB and LS among HIVuninfected children [30]. Similarly, in a smaller study of 66 perinatally HIV-infected children in The Netherlands (mean age 6.7 years) who were mainly receiving a nelfinavir-containing regimen, $8 \%$ had low LS BMD [46]. In these studies we did not observe consistent patterns with respect to sex, race, age group, or anatomic site and prevalence of low BMD.

\begin{tabular}{|c|c|c|c|c|c|c|c|c|c|}
\hline $\begin{array}{l}\text { Author } \\
\text { name } \\
\text { (year) } \\
\text { Ref }\end{array}$ & Country & $\begin{array}{l}\text { Study } \\
\text { design }\end{array}$ & $\begin{array}{l}\text { Comparison } \\
\text { source }\end{array}$ & $\begin{array}{l}\mathrm{N} \\
\text { HIV+/Control/ } \\
\text { Total }\end{array}$ & $\begin{array}{l}\text { HIV+ } \\
\text { sex } \\
(\% \mathrm{M})\end{array}$ & $\begin{array}{l}\text { HIV+ } \\
\text { Age (years) }\end{array}$ & $\begin{array}{l}\mathrm{HIV+} \\
\text { race/ } \\
\text { ethnicity }\end{array}$ & Measurements & Key Findings \\
\hline $\begin{array}{l}\text { Mora } \\
(2001) \\
{[33]}\end{array}$ & Italy & $\begin{array}{l}\text { Cross } \\
\text { sectional }\end{array}$ & $\begin{array}{l}\text { Recruited } \\
\text { healthy } \\
\text { control group } \\
(\mathrm{N}=314)\end{array}$ & $40 / 314 / 354$ & 45 & $\begin{array}{l}\text { Range: } \\
6-17\end{array}$ & $100 \% \mathrm{~W}$ & $\begin{array}{l}\text { DXA (Lunar): WB } \\
\text { BMD, LS BMD }\end{array}$ & $\begin{array}{l}\text { Children on ART had lower WB } \\
\text { BMD, LS BMD compared to } \\
\text { control, adjusted for sex, age, } \\
\text { bone area, weight, and height. } \\
\text { WB BMD was lower in children } \\
\text { on ART with lipodystrophy than } \\
\text { children not on treatment }\end{array}$ \\
\hline $\begin{array}{l}\text { O'Brien } \\
(2001) \\
{[21]}\end{array}$ & USA & $\begin{array}{l}\text { Cross } \\
\text { sectional }\end{array}$ & 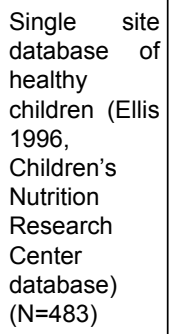 & $19 / 483 / 502$ & 0 & $\begin{array}{l}\text { Mean (SD): } \\
9.2(2.6) \\
\text { Range: } \\
5.9-15.2\end{array}$ & $\begin{array}{l}89 \% \text { B } \\
11 \% \text { W }\end{array}$ & $\begin{array}{l}\text { DXA (Hologic): WB } \\
\text { BMC, WB BMD }\end{array}$ & $\begin{array}{l}\text { Lower WB BMC compared to } \\
\text { control (age, race, and height } \\
\text { matched). } \\
\text { Low dietary calcium intake and } \\
\text { elevated renal excretion. } \\
\text { Calcitropic hormone (1,25 OH } \\
\text { vitamin D and PTH) alterations } \\
\text { related to increased bone } \\
\text { resorption (NTx) }\end{array}$ \\
\hline $\begin{array}{l}\text { Arpadi } \\
(2002) \\
{[22]}\end{array}$ & USA & $\begin{array}{l}\text { Cross } \\
\text { sectional }\end{array}$ & $\begin{array}{l}\text { HIV- } \\
\text { uninfected } \\
\text { children } \\
\text { enrolled in } \\
\text { body } \\
\text { composition } \\
\text { study at the } \\
\text { same site } \\
(\mathrm{N}=262)\end{array}$ & $51 / 262 / 313$ & 51 & $\begin{array}{l}\text { Range: } \\
4.2-14.7\end{array}$ & $\begin{array}{l}51 \% \mathrm{H} \\
41.5 \% \mathrm{~B} \\
7.5 \% \mathrm{~W}\end{array}$ & $\begin{array}{l}\text { DXA } \\
\text { BMC }\end{array}$ (Lunar): WB & $\begin{array}{l}\text { Lower WB BMC compared to } \\
\text { controls, adjusted for age, sex, } \\
\text { race, height and weight. } \\
\text { Magnitude of difference between } \\
\text { HIV+ and controls increased with } \\
\text { age }\end{array}$ \\
\hline $\begin{array}{l}\text { Gaughan } \\
(2002) \\
{[32]}\end{array}$ & USA & $\begin{array}{l}\text { Longitudin } \\
\text { al }\end{array}$ & $\begin{array}{l}\text { Exposed but } \\
\text { uninfected } \\
\text { children } \\
(\mathrm{N}=849)\end{array}$ & $2014 / 849 / 2863$ & 50 & $\begin{array}{l}\text { Median: } 5.3 \\
\text { 10th, } 90 \text { th: } \\
1,12\end{array}$ & $\begin{array}{l}16 \% \mathrm{~W} \\
52 \% \mathrm{~B} \\
31 \% \mathrm{H} \\
1 \% \mathrm{O}\end{array}$ & $\begin{array}{l}\text { Legg-Calve-Perthes } \\
\text { disease (LCPD) }\end{array}$ & $\begin{array}{l}\text { Six cases of LCPD including } 4 \\
\text { cases reported at study entry and } \\
2 \text { diagnosed during } 5837 \text { person- } \\
\text { years of follow-up }\end{array}$ \\
\hline $\begin{array}{l}\text { Zamboni } \\
(2003) \\
{[34]}\end{array}$ & Italy & $\begin{array}{l}\text { Cross } \\
\text { sectional }\end{array}$ & $\begin{array}{l}\text { Normal } \\
\text { prepubertal }\end{array}$ & $13 / 198 / 211$ & 31 & $\begin{array}{l}\text { Range: } \\
4-12\end{array}$ & $\begin{array}{l}\text { Not } \\
\text { specified }\end{array}$ & $\begin{array}{lr}\text { DXA } & \text { (Lunar): } \quad \text { LS } \\
\text { BMD, } & \text { calculated } \\
\text { vBMD } & \end{array}$ & $\begin{array}{l}\text { Densitometry results not } \\
\text { compared with controls }\end{array}$ \\
\hline
\end{tabular}


Citation: Arpadi SM, Shiau S, Arpadi CM, Yin MT (2014) Bone Health in HIV-Infected Children, Adolescents and Young Adults: A Systematic

\begin{tabular}{|c|c|c|c|c|c|c|c|c|c|}
\hline & & & $\begin{array}{l}\text { population } \\
(\mathrm{N}=198)\end{array}$ & & & & & & $\begin{array}{l}\text { Negative VBMD Z-score (Z-score } \\
<0.0 \text { ) in } 6 / 13 \\
\text { Spontaneous clavicular fracture } \\
\text { in } 1 \text { child with low VBMD z-score } \\
\text { Low bone formation and high } \\
\text { bone resorption markers (e.g. low } \\
\text { OCN, high NTX, low IGF in } \\
\text { children with low CD4 and high } \\
\text { IL-6) }\end{array}$ \\
\hline $\begin{array}{l}\text { Mora } \\
(2004) \\
{[35]}\end{array}$ & Italy & $\begin{array}{l}\text { Longitudin } \\
\text { al }\end{array}$ & $\begin{array}{l}\text { Recruited } \\
\text { control group } \\
\text { of healthy } \\
\text { volunteers of } \\
\text { comparable } \\
\text { ages }(\mathrm{N}=381)\end{array}$ & $32 / 381 / 413$ & 53 & $\begin{array}{l}\text { Mean (SD): } \\
12.4(0.5) \\
\text { Range: } \\
6.3-17.7\end{array}$ & $100 \% \mathrm{~W}$ & $\begin{array}{l}\text { DXA (Lunar): WB } \\
\text { BMD, LS BMD }\end{array}$ & $\begin{array}{l}\text { Lower WB BMD and LS BMD } \\
\text { compared to control at baseline, } \\
\text { adjusted for sex, age, Tanner } \\
\text { stage, and weight } \\
\text { Lower annual increment for WB } \\
\text { BMD compared to control } \\
\text { Higher bone turnover rate } \\
\text { compared to controls (BSAP and } \\
\text { NTx) }\end{array}$ \\
\hline $\begin{array}{l}\text { Stagi } \\
(2004) \\
{[36]}\end{array}$ & Italy & $\begin{array}{l}\text { Cross- } \\
\text { sectional }\end{array}$ & $\begin{array}{l}\text { Recruited } \\
\text { control group } \\
\text { matched by } \\
\text { age, pubertal } \\
\text { stage, and } \\
\text { sex ( } \mathrm{N}=55) \\
\text { and also } \\
\text { used } \\
\text { normative } \\
\text { database } \\
\text { (CUBA for } \\
\text { age } 5-15 \\
\text { years, Falcini } \\
2003 \text { for } \\
\text { under age } 5 \\
\text { years) }\end{array}$ & $44 / 55 / 99$ & 48 & $\begin{array}{l}\text { Median: } 8.4 \\
\text { Range: } \\
4.6-12.4\end{array}$ & $100 \% \mathrm{~W}$ & $\begin{array}{l}\text { QUS (McCue } \\
\text { Ultrasonics): heel BUA }\end{array}$ & $\begin{array}{l}\text { Children with severe clinical } \\
\text { symptoms had lower BUA } \\
\text { compared to controls (age, sex, } \\
\text { and pubertal-stage sex matched) } \\
\text { BUA related to free IGF-1 }\end{array}$ \\
\hline $\begin{array}{l}\text { Giacomet } \\
(2005) \\
{[37]}\end{array}$ & Italy & $\begin{array}{l}\text { Longitudin } \\
\text { al }\end{array}$ & $\begin{array}{l}\text { Recruited } \\
\text { control group } \\
\text { of healthy } \\
\text { white } \\
\text { volunteers } \\
(\mathrm{N}=166)\end{array}$ & $16 / 166 / 182$ & $\begin{array}{l}\text { Not } \\
\text { reported }\end{array}$ & $\begin{array}{l}\text { Mean } 13.3 \\
\text { Range } \\
6.4-17.9\end{array}$ & $100 \% \mathrm{~W}$ & $\begin{array}{l}\text { DXA (Lunar): LS } \\
\text { BMC, LS BMD, WB } \\
\text { BMC, WB BMD }\end{array}$ & $\begin{array}{l}\text { During treatment with tenofovir, } \\
\text { WB BMC, WB BMD, LS BMC, LS } \\
\text { BMD changes did not differ from } \\
\text { expected }\end{array}$ \\
\hline $\begin{array}{l}\text { Hazra } \\
(2005) \\
{[23]}\end{array}$ & USA & $\begin{array}{l}\text { Longitudin } \\
\text { al }\end{array}$ & $\begin{array}{l}\text { Databases } \\
\text { Bachrach } \\
1999, \\
\text { Faulkner } \\
1996\end{array}$ & 18/NS/18 & 61 & $\begin{array}{l}\text { Mean (SD): } \\
12(2.5) \\
\text { Range: } \\
8.3-16.2\end{array}$ & $\begin{array}{l}33 \% \mathrm{~W} \\
55 \% \mathrm{~B} \\
6 \% \mathrm{H} \\
6 \% \mathrm{O}\end{array}$ & $\begin{array}{ll}\text { DXA } & \text { (Hologic): LS } \\
\text { BMD } & \end{array}$ & $\begin{array}{l}\text { Baseline median LS BMD z-score } \\
\text { was }-1.18 \text {, adjusted for age, } \\
\text { ethnicity, and sex } \\
5 \text { of } 15 \text { subjects experienced } \\
\text { decrease in LS BMD at week } 48 \\
\text { after starting tenofovir }\end{array}$ \\
\hline $\begin{array}{l}\text { Jacobson } \\
(2005) \\
{[24]}\end{array}$ & USA & $\begin{array}{l}\text { Longitudin } \\
\text { al }\end{array}$ & $\begin{array}{l}\text { Siblings } \\
(\mathrm{N}=9) \text { and } \\
\text { single site } \\
\text { multiethnic } \\
\text { database } \\
\text { (Ellis 2001, } \\
\text { Children's } \\
\text { Nutrition } \\
\text { Research } \\
\text { Center } \\
\text { database) }\end{array}$ & $37 / 9 / 46$ & 49 & $\begin{array}{l}\text { Median: } \\
11.6 \\
\text { Range: } \\
9.6-13.8\end{array}$ & $\begin{array}{l}40 \% \mathrm{~B} \\
27 \% \mathrm{H} \\
24 \% \mathrm{~W}\end{array}$ & $\begin{array}{l}\text { DXA (Lunar or } \\
\text { Hologic): WB BMC, } \\
\text { WB BMD }\end{array}$ & $\begin{array}{l}\text { Lower WB BMD than population } \\
\text { but not in comparison to sibling } \\
\text { controls, adjusted for height and } \\
\text { weight } z \text {-scores } \\
\text { All controls had stable or } \\
\text { increased repeat WB BMD but } \\
\text { only } 44 \% \text { of HIV+ }(p=0.09)\end{array}$ \\
\hline $\begin{array}{l}\text { Mora } \\
(2005) \\
{[38]}\end{array}$ & Italy & $\begin{array}{l}\text { Cross- } \\
\text { sectional }\end{array}$ & $\begin{array}{l}\text { Recruited } \\
\text { control group } \\
\text { of healthy } \\
\text { children } \\
(\mathrm{N}=119)\end{array}$ & 16/119/135 & 38 & $\begin{array}{l}\text { Mean (SD): } \\
9.3(3.9) \\
\text { Range: } \\
4.4-16.0\end{array}$ & $100 \% \mathrm{~W}$ & $\begin{array}{l}\text { DXA (Lunar): LS BMC } \\
\text { \& WB BMC }\end{array}$ & $\begin{array}{l}\text { LS BMC and WB BMC not } \\
\text { significantly lower than controls, } \\
\text { adjusted for sex, weight, and } \\
\text { bone area }\end{array}$ \\
\hline
\end{tabular}


Citation: $\quad$ Arpadi SM, Shiau S, Arpadi CM, Yin MT (2014) Bone Health in HIV-Infected Children, Adolescents and Young Adults: A Systematic

\begin{tabular}{|c|c|c|c|c|c|c|c|c|c|}
\hline $\begin{array}{l}\text { Pitukche } \\
\text { ewanont } \\
(2005) \\
{[25]}\end{array}$ & USA & $\begin{array}{l}\text { Cross } \\
\text { sectional }\end{array}$ & $\begin{array}{l}\text { Recruited } \\
\text { control group } \\
\text { matched for } \\
\text { age, gender, } \\
\text { ethnicity } \\
(\mathrm{N}=58)\end{array}$ & $58 / 58 / 116$ & 45 & $\begin{array}{l}\text { Mean (SD): } \\
12.03 \\
(3.88) \\
\text { Range: } \\
5-19.39\end{array}$ & $\begin{array}{l}\text { "of multiple } \\
\text { ethnicities" }\end{array}$ & $\begin{array}{l}\text { DXA (Hologic): LS } \\
\text { bone area, LS BMC, } \\
\text { LS BMD, WB bone } \\
\text { area, WB BMC, WB } \\
\text { BMD } \\
\text { QCT (General Electric } \\
\text { Hilite Advantage): } \\
\text { vertebral BD, vertebral } \\
\text { height, vertebral CSA }\end{array}$ & $\begin{array}{l}\text { Less LS bone area, LS BMC, LS } \\
\text { BMD, WB bone area, WB BMC, } \\
\text { WB BMD compared to controls } \\
\text { (unadjusted). } \\
\text { Variance in bone area, BMC, and } \\
\text { BMD largely accounted for by } \\
\text { height and weight } \\
\text { Similar volumetric BMD by QCT } \\
\text { in HIV+ compared to controls but } \\
\text { smaller vertebral height and CSA }\end{array}$ \\
\hline $\begin{array}{l}\text { Rosso } \\
(2005) \\
{[39]}\end{array}$ & Italy & $\begin{array}{l}\text { Cross } \\
\text { sectional }\end{array}$ & $\begin{array}{l}\text { Recruited } \\
\text { control group } \\
\text { from schools } \\
(\mathrm{N}=1227)\end{array}$ & $44 / 1227 / 1271$ & 48 & $\begin{array}{l}\text { Median: } \\
10.7 \\
\text { Mean (SD): } \\
10.4(4.0) \\
\text { Range: } \\
\text { 3-17 }\end{array}$ & $100 \% \mathrm{~W}$ & $\begin{array}{l}\text { QUS: } \quad \text { phalangeal } \\
\text { SOS, BTT }\end{array}$ & $\begin{array}{l}\text { Lower SOS and BTT compared } \\
\text { to control, adjusted for age, bone } \\
\text { age, and body size }\end{array}$ \\
\hline $\begin{array}{l}\text { Gafni } \\
(2006) \\
{[26]}\end{array}$ & USA & $\begin{array}{l}\text { Longitudin } \\
\text { al }\end{array}$ & $\begin{array}{l}\text { For children } \\
>9 \text { years: } \\
\text { single site } \\
\text { multi-ethnic } \\
\text { longitudinal } \\
\text { database } \\
\text { ( } \mathrm{N}=423) \\
\text { (Bachrach } \\
\text { 1999) } \\
\text { For children } \\
<8 \text { years old: } \\
\text { single site } \\
\text { longitudinal } \\
\text { database } \\
\text { (Faulkner } \\
\text { 1996) }\end{array}$ & 15/NS/15 & 67 & $\begin{array}{l}\text { Range: } \\
4-18\end{array}$ & NS & $\begin{array}{lll}\text { DXA } & \text { (Hologic): } & \text { LS } \\
\text { BMD, } & \text { FN BMD, } & \text { TH } \\
\text { BMD, LS BMAD } & \end{array}$ & $\begin{array}{l}\text { Baseline median z-scores were: } \\
\text { LS BMD -1.2, TH BMD -1.0, FN } \\
\text { BMD -1.4, LS BMAD }-0.9 \\
\text { Decrease in BMD and BMD z- } \\
\text { score at LS, FN, and TH from } \\
\text { baseline to } 24 \text { weeks and } 48 \\
\text { weeks after tenofovir initiation } \\
\text { and then stabilized }\end{array}$ \\
\hline $\begin{array}{l}\text { Mora } \\
(2007) \\
{[40]}\end{array}$ & Italy & $\begin{array}{l}\text { Longitudin } \\
\text { al }\end{array}$ & $\begin{array}{l}\text { Control group } \\
\text { of healthy } \\
\text { children } \\
(\mathrm{N}=336)\end{array}$ & $27 / 336 / 363$ & 48 & $\begin{array}{l}\text { Range: } \\
4.9-17.3\end{array}$ & $100 \% \mathrm{~W}$ & $\begin{array}{l}\text { DXA (Lunar): LS } \\
\text { BMD, WB BMD }\end{array}$ & $\begin{array}{l}\text { Lower WB BMD and LS BMD } \\
\text { compared to controls, adjusted } \\
\text { for sex, age, weight, and height }\end{array}$ \\
\hline $\begin{array}{l}\text { Purdy } \\
(2008) \\
{[27]}\end{array}$ & USA & $\begin{array}{l}\text { Longitudin } \\
\text { al }\end{array}$ & None & $6 / N A / 6$ & 67 & $\begin{array}{l}\text { Median: } \\
12.8 \\
\text { Range: } \\
11.3-17.5\end{array}$ & N/A & $\begin{array}{ll}\text { DXA } & \text { (Hologic): LS } \\
\text { BMD } & \end{array}$ & $\begin{array}{l}5 / 6 \text { children had decreases in LS } \\
\text { BMD after receiving tenofovir as } \\
\text { part of new regimen }\end{array}$ \\
\hline $\begin{array}{l}\text { Mora } \\
(2009) \\
{[41]}\end{array}$ & Italy & $\begin{array}{l}\text { Cross } \\
\text { sectional }\end{array}$ & $\begin{array}{l}\text { Manufacturer } \\
\text { 's software } \\
\text { (DXA: DPX-L } \\
\text { version, } \\
\text { version } \quad 1.5 ; \\
\text { QUS: } \\
\text { BeamMed) }\end{array}$ & $88 / \mathrm{NS} / 88$ & 49 & $\begin{array}{l}\text { Range: } \\
4.8-22.1\end{array}$ & $\begin{array}{l}78 / 88 \mathrm{~W} \\
10 / 88 \mathrm{~B}\end{array}$ & $\begin{array}{l}\text { DXA (Lunar): LS BMC } \\
\& \text { BMD, WB BMC \& } \\
\text { BMD } \\
\text { QUS (BeamMed): } \\
\text { tibia, radius SOS }\end{array}$ & $\begin{array}{l}\text { SoS associated with LS BMC, LS } \\
\text { BMD, WB BMC, WB BMD, } \\
\text { adjusted for sex, weight, and } \\
\text { height }\end{array}$ \\
\hline $\begin{array}{l}\text { Jacobson } \\
(2010)[6]\end{array}$ & USA & $\begin{array}{l}\text { Cross } \\
\text { sectional }\end{array}$ & $\begin{array}{l}\text { Recruited } \\
\text { control group } \\
\text { of uninfected } \\
\text { children into } \\
3 \text { Tanner } \\
\text { strata with } \\
\text { similar overall } \\
\text { distribution } \\
\text { for sex and } \\
\text { race/ethnicity } \\
\text { as the HIV+ }\end{array}$ & $236 / 143 / 379$ & 53 & $\begin{array}{l}\text { Median: } \\
12.6 \\
\text { Range: } \\
7-24\end{array}$ & $\begin{array}{l}13.1 \% \mathrm{~W} \\
54.7 \% \mathrm{~B} \\
32.2 \% \mathrm{H}\end{array}$ & $\begin{array}{llll}\text { DXA } & \text { (Lunar } & \text { or } \\
\text { Hologic): LS } & \text { BMC } & \& \\
\text { BMD, WB } & \text { BMC } & \& \\
\text { BMD } & & & \end{array}$ & $\begin{array}{l}\text { HIV-infected males had lower WB } \\
\text { BMC and WB BMD and LS BMD } \\
\text { at Tanner } 5 \text { than controls, } \\
\text { adjusted for DXA scanner, race, } \\
\text { HIV, Tanner group, interaction of } \\
\text { HIV and Tanner group, age, } \\
\text { height, lean body mass. No } \\
\text { differences in girls } \\
\text { Use of NNRTI associated with } \\
\text { higher LS BMC and LS BMD } \\
\text { Ritonavir-boosted protease } \\
\text { inhibitors associated with lower } \\
\text { BMC and WB and LS BMD } \\
\text { No effect of cumulative time on } \\
\text { ART and bone measures }\end{array}$ \\
\hline
\end{tabular}


Citation: Arpadi SM, Shiau S, Arpadi CM, Yin MT (2014) Bone Health in HIV-Infected Children, Adolescents and Young Adults: A Systematic

Page 6 of 14

\begin{tabular}{|c|c|c|c|c|c|c|c|c|c|}
\hline & & & & & & & & & $\begin{array}{l}\text { Conclusions with spinal BMAD } \\
\text { same as LS BMD }\end{array}$ \\
\hline $\begin{array}{l}\text { Rosso } \\
(2010) \\
{[42]}\end{array}$ & Italy & $\begin{array}{l}\text { Longitudin } \\
\text { al }\end{array}$ & $\begin{array}{l}\text { Manufacturer } \\
\text { software (not } \\
\text { specified) }\end{array}$ & 8/NS/8 & 75 & $\begin{array}{l}\text { Median: } \\
11.2 \\
\text { Range: } \\
3.8-18.2\end{array}$ & NS & $\begin{array}{l}\text { QUS (not specified): } \\
\text { SOS and BTT }\end{array}$ & $\begin{array}{l}\text { SOS and BTT z-score values did } \\
\text { not show differences during } \\
\text { tenofovir treatment }\end{array}$ \\
\hline $\begin{array}{l}\text { Vigano } \\
(2010) \\
{[43]}\end{array}$ & Italy & $\begin{array}{l}\text { Longitudin } \\
\text { al }\end{array}$ & $\begin{array}{l}\text { Manufacturer } \\
\text { software } \\
\text { (enCORE } \\
\text { software, } \\
\text { version 13, } \\
\text { GE medical } \\
\text { systems) }\end{array}$ & $21 / N S / 21$ & 48 & $\begin{array}{l}\text { At baseline: } \\
\text { Median: } \\
12.1 \\
\text { Range: } \\
4.9-17.9\end{array}$ & $100 \% \mathrm{~W}$ & $\begin{array}{l}\text { DXA (Lunar): WB } \\
\text { BMD and LS BMD }\end{array}$ & $\begin{array}{l}\text { LS BMD and WB BMD did not } \\
\text { change during } 60 \text { month period } \\
\text { after starting tenofovir treatment }\end{array}$ \\
\hline $\begin{array}{l}\text { Zuccotti } \\
(2010) \\
{[44]}\end{array}$ & Italy & $\begin{array}{l}\text { Cross } \\
\text { sectional }\end{array}$ & $\begin{array}{l}\text { Recruited } \\
\text { healthy } \\
\text { controls of } \\
\text { comparable } \\
\text { age }(\mathrm{N}=194)\end{array}$ & $86 / 194 / 280$ & 45 & $\begin{array}{l}\text { Range: } \\
4.8-22.1\end{array}$ & NS & $\begin{array}{l}\text { DXA (Lunar): LS BMC } \\
\& \text { BMD, WB BMC \& } \\
\text { BMD }\end{array}$ & $\begin{array}{l}\text { Lower WB BMC, LS BMC and } \\
\text { BMD in children receiving PI- } \\
\text { based treatment compared to } \\
\text { healthy } \\
\text { LS BMC lower in children } \\
\text { receiving stavudine or ritonavir } \\
\text { compared to healthy and } \\
\text { treatment naïve or those on other } \\
\text { ART. } \\
\text { Lower WB BMD in children on } \\
\text { ART compared to healthy } \\
\text { No differences between ART } \\
\text { naïve and healthy children } \\
\text { All models adjusted for age, sex, } \\
\text { pubertal stage, weight, height }\end{array}$ \\
\hline $\begin{array}{l}\text { Arpadi } \\
(2012) \\
{[28]}\end{array}$ & USA & $\begin{array}{l}\text { Longitudin } \\
\text { al }\end{array}$ & $\begin{array}{l}\text { Multicenter } \\
\text { study of } \\
\text { healthy } \\
\text { children } \\
\text { (Bone } \\
\text { Mineral } \\
\text { Density of } \\
\text { Childhood } \\
\text { Study } \\
\text { Kalkwarf } \\
\text { 2007) }\end{array}$ & $59 / N S / 59$ & NS & $\begin{array}{l}\text { Range: } \\
6-16\end{array}$ & $\begin{array}{l}63 \% \mathrm{~B} \\
37 \% \mathrm{H}\end{array}$ & $\begin{array}{l}\text { DXA (Hologic): WB } \\
\text { BMC and WB BMD }\end{array}$ & $\begin{array}{l}\text { WB BMC WB BMD LS BMC and } \\
\text { LS BMD increased at } 1 \text { year and } \\
2 \text { year for both randomized } \\
\text { groups in the study. No group } \\
\text { differences. }\end{array}$ \\
\hline $\begin{array}{l}\text { Della } \\
\text { Negra } \\
(2012) \\
{[50]}\end{array}$ & $\begin{array}{l}\text { USA, } \\
\text { Brazil, } \\
\text { Panam } \\
\text { a }\end{array}$ & $\begin{array}{l}\text { Longitudin } \\
\text { al }\end{array}$ & $\begin{array}{l}\text { Manufacturer } \\
\text { software (not } \\
\text { specified but } \\
\text { used age- } \\
\text { matched, } \\
\text { sex-matched, } \\
\text { and race- } \\
\text { matched } \\
\text { healthy } \\
\text { controls) }\end{array}$ & $90 / \mathrm{NS} / 90$ & 44 & $\begin{array}{l}\text { Mean (SD): } \\
14(1.5)\end{array}$ & $100 \% \mathrm{H}$ & $\begin{array}{l}\text { DXA (Lunar } r \\
\text { Hologic): } \\
\text { WB BMD }\end{array}$ & $\begin{array}{l}\text { Median WB BMD Z-score } \\
\text { decreased }-0.2 \text { and LS BMD Z- } \\
\text { score decreased }-0.2 \text { for TDF } \\
\text { group and WB BMD Z-score }-0.1 \\
\text { and LS BMD Z-score }-0.1 \text { for } \\
\text { placebo group in first } 24 \text { weeks }\end{array}$ \\
\hline $\begin{array}{l}\text { Mulligan } \\
(2012)[7]\end{array}$ & USA & $\begin{array}{l}\text { Cross } \\
\text { sectional }\end{array}$ & $\begin{array}{l}\text { Recruited } \\
\text { control group } \\
\text { of } \\
\text { seronegative } \\
\text { men from the } \\
\text { same age } \\
\text { range at } \\
\text { same sites as } \\
\mathrm{HIV}+(\mathrm{N}=53)\end{array}$ & $199 / 53 / 252$ & 100 & $\begin{array}{l}\text { Median: } 21 \\
\text { Range } \\
14-25\end{array}$ & $\begin{array}{l}59.8 \% \mathrm{~B} \\
28.1 \% \mathrm{H} \\
12.1 \% \mathrm{O}\end{array}$ & $\begin{array}{l}\text { DXA (Lunar or } \\
\text { Hologic): TH BMD, LS } \\
\text { BMD, WB BMD, TH } \\
\text { BMC, LS BMC, WB } \\
\text { BMC }\end{array}$ & $\begin{array}{l}\text { WB BMD and WB BMC Z-scores } \\
\text { lower among HIV-infected on } \\
\text { ART, in particular for those on PI, } \\
\text { compared to controls, adjusted } \\
\text { for race, BMI, type of DXA } \\
\text { scanner } \\
\text { FN BMD and FN BMD Z-scores } \\
\text { only lower for those on PI } \\
\text { compared to ART-naïve HIV+ } \\
\text { and controls }\end{array}$ \\
\hline $\begin{array}{l}\text { Puthanak } \\
\text { it } \\
(2012) \\
{[47]}\end{array}$ & $\begin{array}{l}\text { Thailan } \\
\text { d }\end{array}$ & $\begin{array}{l}\text { Cross } \\
\text { sectional }\end{array}$ & $\begin{array}{l}\text { Data from } \\
\text { cohort of } 199 \\
\text { HIV- } \\
\text { uninfected }\end{array}$ & 101/199/300 & 51 & $\begin{array}{l}\text { Median: } \\
14.3 \\
\text { IQR: } \\
\text { 13.0-15.7 }\end{array}$ & $100 \%$ Thai & $\begin{array}{ll}\text { DXA (Lunar } & \text { or } \\
\text { Hologic): LS BMD } & \end{array}$ & $\begin{array}{l}24 \% \text { of HIV-infected have low LS } \\
\text { BMD ( } \mathrm{Z} \text { score } \leq-2) \\
\text { LS BMD lower compared to } \\
\text { controls (age and sex-matched) }\end{array}$ \\
\hline
\end{tabular}


Citation: Arpadi SM, Shiau S, Arpadi CM, Yin MT (2014) Bone Health in HIV-Infected Children, Adolescents and Young Adults: A Systematic

\begin{tabular}{|c|c|c|c|c|c|c|c|c|c|}
\hline & & & $\begin{array}{l}\text { children aged } \\
\text { from } \\
12-18 \text { years } \\
(N=199)\end{array}$ & & & & & & $\begin{array}{l}\text { Advanced clinical disease prior to } \\
\text { initiation of ART and short stature } \\
\text { associated with low LS BMD }\end{array}$ \\
\hline $\begin{array}{l}\text { Siberry } \\
(2012) \\
{[29]}\end{array}$ & USA & $\begin{array}{l}\text { Longitudin } \\
\text { al }\end{array}$ & $\begin{array}{l}\text { Exposed but } \\
\text { uninfected } \\
\text { children }\end{array}$ & 1326/649/1975 & 49 & $\begin{array}{l}\text { Mean: } 7.1 \\
\text { Range: } 5.0 \text {, } \\
10.0\end{array}$ & $\begin{array}{l}62 \% \mathrm{~B} \\
11 \% \mathrm{~W} \\
24 \% \mathrm{H} \\
2 \% \mathrm{O}\end{array}$ & Fractures & $\begin{array}{l}\text { No increased risk of fracture in } \\
\text { HIV-infected compared to HIV- } \\
\text { exposed but uninfected children }\end{array}$ \\
\hline $\begin{array}{l}\text { Schtsche } \\
\text { rbyna } \\
(2012) \\
{[49]}\end{array}$ & Brazil & $\begin{array}{l}\text { Cross } \\
\text { sectional }\end{array}$ & $\begin{array}{l}\text { Manufacturer } \\
\text { software } \\
\text { (Prodigy } \\
\text { software } \\
\text { version 11.4) }\end{array}$ & 74/NS/74 & 45 & $\begin{array}{l}\text { Mean (SD): } \\
17.3(1.8)\end{array}$ & $\begin{array}{l}36.5 \% \mathrm{~W} \\
63.5 \% \text { not } \\
\mathrm{W}\end{array}$ & $\begin{array}{l}\text { DXA (Lunar) WB, LS } \\
\text { BMD }\end{array}$ & $\begin{array}{l}\text { Low WB and/or LS BMD (Z score } \\
<-2 \text { ) in } 32.4 \% \text { of population } \\
\text { Children on tenofovir had lower } \\
\text { LS BMD and WB BMD } \\
\text { Time on TDF inversely correlated } \\
\text { to LS BMD }\end{array}$ \\
\hline $\begin{array}{l}\text { Bunders } \\
(2013) \\
{[46]}\end{array}$ & $\begin{array}{l}\text { Netherl } \\
\text { ands }\end{array}$ & $\begin{array}{l}\text { Longitudin } \\
\text { al }\end{array}$ & $\begin{array}{l}\text { Manufacturer } \\
\text { software } \\
\text { (unspecified) }\end{array}$ & $66 / N S / 66$ & 45 & $\begin{array}{l}\text { Median: } 6.7 \\
\text { IQR: } \\
4.4-10.3\end{array}$ & $62 \%$ B & $\begin{array}{l}\text { DXA (Hologic): LS } \\
\text { BMD, left FN BMD }\end{array}$ & $\begin{array}{l}\text { Low LS BMD ( } Z \text { score }<-2.0) \text { in } \\
8 \% \text { of HIV+ children } \\
\text { Lower LS BMD compared to } \\
\text { controls at baseline; median LS } \\
\text { BMD } z \text {-score was }-0.9\end{array}$ \\
\hline $\begin{array}{l}\text { DiMeglio } \\
(2013) \\
{[30]}\end{array}$ & $\begin{array}{l}\text { USA } \\
\text { and } \\
\text { Puerto } \\
\text { Rico }\end{array}$ & $\begin{array}{l}\text { Cross } \\
\text { sectional }\end{array}$ & $\begin{array}{l}\text { Exposed } \\
\text { uninfected } \\
\text { children } \\
(\mathrm{N}=160)\end{array}$ & $350 / 160 / 510$ & 46 & $\begin{array}{l}\text { Median: } \\
12.6 \\
\text { IQR: } 10.2, \\
14.4\end{array}$ & $\begin{array}{l}26 \% \mathrm{H} \\
66 \% \mathrm{~B} \\
8 \% \mathrm{O} / \mathrm{W}\end{array}$ & $\begin{array}{l}\text { DXA (Lunar } r \\
\text { Hologic): } \\
\text { LS BMD }\end{array}$ & $\begin{array}{l}\text { Lower TB and LS BMD Z-scores } \\
\text { adjusted for sex, race/ethnicity } \\
\text { and puberty stage compared to } \\
\text { control } \\
\text { No differences with further } \\
\text { adjustment for weight and height. } \\
\text { WB BMD lower with use of } \\
\text { lamivudine and ritonavir-boosted } \\
\text { protease inhibitor }\end{array}$ \\
\hline $\begin{array}{l}\text { Lima } \\
(2013) \\
{[48]}\end{array}$ & Brazil & $\begin{array}{l}\text { Cross } \\
\text { sectional }\end{array}$ & $\begin{array}{l}\text { Database } \\
\text { from } \\
\text { NHANES } \\
\text { (Kelly 2009) }\end{array}$ & $48 / N S / 48$ & 50 & $\begin{array}{l}\text { Mean (SD): } \\
12.7(2.7) \\
\text { Range: } \\
7-17\end{array}$ & $\begin{array}{l}53.3 \% \mathrm{~W} \\
43.8 \% \mathrm{~B}\end{array}$ & $\begin{array}{l}\text { DXA (Hologic): WB } \\
\text { BMD, LS BMD }\end{array}$ & $\begin{array}{l}16.7 \% \text { of HIV+ had low bone } \\
\text { mass for age } \\
\text { WB BMD Z-scores greater in } \\
\text { adolescence compared to } \\
\text { children. } \\
\text { No difference by sex once height } \\
\text { accounted for. } \\
\text { Association between ritonavir- } \\
\text { boosted protease inhibitors and } \\
\text { lower WB BMD Z-score }\end{array}$ \\
\hline $\begin{array}{l}\text { MacDona } \\
\text { ld } \\
(2013) \\
{[45]}\end{array}$ & Canada & $\begin{array}{l}\text { Longitudin } \\
\text { al }\end{array}$ & $\begin{array}{l}\text { Healthy } \\
\text { controls } \\
\text { who were } \\
\text { participants in } \\
\text { the University } \\
\text { of British } \\
\text { Columbia } \\
\text { Healthy } \\
\text { Bones III } \\
\text { follow-up } \\
\text { study } \\
(\mathrm{N}=883)\end{array}$ & $31 / 883 / 914$ & 61 & $\begin{array}{l}\text { Median: } \\
13.6 \\
\text { IQR: } 11.6, \\
16.0\end{array}$ & $\begin{array}{l}32.2 \% \\
\text { Mixed } \\
25.8 \% \mathrm{~B} \\
22.6 \% \\
\text { Aboriginal } \\
12.9 \% \mathrm{~W} \\
6.5 \% \mathrm{~A}\end{array}$ & $\begin{array}{l}\text { DXA (Hologic): WB } \\
\text { BMC, LS BMC, femur } \\
\text { BMC } \\
\text { Peripheral QCT } \\
\text { (Norland/Stratec } \\
\text { XCT): muscle CSA, } \\
\text { total and cortical bone } \\
\text { area, cortical BMD, } \\
\text { thickness and strength } \\
\text { strain index at tibial } \\
\text { shaft }\end{array}$ & $\begin{array}{l}\text { WB BMC and femur BMC lower } \\
\text { among HIV+, adjusted for height } \\
\text { and lean mass } \\
\text { CD4\% positively associated with } \\
\text { WB BMD z-score } \\
\text { Muscle CSA lower in HIV+ } \\
\text { Tibial total and cortical bone area } \\
\text { not different than controls. } \\
\text { Cortical BMD positively } \\
\text { associated with NNRTI use } \\
\text { Cortical thickness negatively } \\
\text { associated with PI use }\end{array}$ \\
\hline $\begin{array}{l}\text { Yin } \\
(2014) \\
{[31]}\end{array}$ & USA & $\begin{array}{l}\text { Cross- } \\
\text { sectional }\end{array}$ & $\begin{array}{l}\text { Recruited } \\
\text { control group } \\
\text { of HIV- } \\
\text { uninfected } \\
\text { controls } \\
(\mathrm{N}=15)\end{array}$ & $30 / 15 / 45$ & 100 & $\begin{array}{l}\text { Mean (SD): } \\
22.5(0.3)\end{array}$ & $\begin{array}{l}60 \% \mathrm{~B} \\
40 \% \mathrm{H}\end{array}$ & $\begin{array}{lll}\text { DXA } & \text { (Hologic): } & \text { LS } \\
\text { BMC, LS } & \text { BMD, } & \text { FN } \\
\text { BMC, } & \text { FN } & \text { BMD, TH } \\
\text { BMC, TH BMD, } & \text { R13 } \\
\text { BMC, R13 } & \text { BMD, UD } \\
\text { BMC, UD BMD } & \\
\text { HR peripheral } & \text { QCT } \\
\text { (XtremeCT Scanco): }\end{array}$ & $\begin{array}{l}\text { DXA BMD Z-scores lower at the } \\
\text { LS, TH, and R13 than controls } \\
\text { HR peripheral QCT total and } \\
\text { trabecular vBMD and cortical and } \\
\text { trabecular thickness lower than } \\
\text { controls }\end{array}$ \\
\hline
\end{tabular}




\begin{tabular}{|l|l|l|l|l|l|l|l|}
\hline & & & & & & $\begin{array}{l}\text { radius CSA, radius } \\
\text { vBMD, radius } \\
\text { microarchitecture, tibia } \\
\text { CSA, tibia vBMD, tibia } \\
\text { microarchitecture }\end{array}$ \\
\hline
\end{tabular}

Table 2: Results of the systematic review on bone health in HIV-infected children, adolescents, and young adults

Reflecting the epidemiology, there are few studies of BMD in children and adolescents with HIV infection acquired by means other than mother-to-child transmission $[7,31,38]$. In a study of 16 ARTnaïve children and adolescents (mean age $9.3 \pm 3.9$ years) with recently acquired HIV (mean duration of infection 2.8 years) due to contaminated blood products, no differences in LS or WB BMC were observed compared to controls, adjusted for sex, weight, and bone area [38]. HIV acquired via sexual transmission during adolescence or young adulthood may have severe impact on bone integrity. DXA Zscores were lower and bone microarchitecture by high resolution peripheral QCT of the radius and tibia was abnormal in young men ages 20-25 who were infected during adolescence than uninfected controls [31]. BMD and microarchitectural indices in these adolescence-infected men were similar to those of perinatally-infected men despite marked differences in the duration of HIV infection and ART exposure [31]. In addition, Mulligan et al. reported on 199 young men (ages 14-25 years) soon after acquiring HIV infection by sexual transmission, finding lower WB BMC Z-scores among those on ART [7].

Bone mass decrements among those with perinatally acquired HIV may become more pronounced with increasing age and biologic maturation $[6,22,24]$ although this was not a consistent finding [8]. Initial studies suggest that peak bone mass, the maximum amount of bony tissue at the end of skeletal maturation is lower among those who acquire HIV perinatally or during adolescence [31].

\section{Calculated volumetric BMD from DXA (BMAD)}

Three of the published studies estimated the LS volumetric BMD from DXA output, with each applying a different method for calculating vertebral body volume $[6,26,34]$. This reflects the lack of consensus on assumptions regarding underlying bone geometry, making it difficult to directly compare results. Calculated volumetric BMD derived by dividing the DXA-measured LS BMC obtained by the lateral scan by the body vertebral volume, was found by Zamboni et al. to be reduced in 6 out of 13 patients (mean age 7.8 years); however, the source of comparison was not specified [34]. When compared to longitudinal data obtained in healthy Canadian children, BMAD calculated as described by Carter et al. [LS BMC/(LS area)1.5] among 15 HIV-infected children of unspecified race was reduced (median -0.9, range -3.2-12) [26,54]. Jacobson et al. used the formula by Katzman et al. [55] to estimate LS BMAD, and the findings and conclusions were the same as those with LS BMD (Table 2) [6].

\section{Measured volumetric BMD}

Studies that directly measured volumetric BMD $\left(\mathrm{g} / \mathrm{cm}^{3}\right)$ had variable findings. Pitukcheewanont et al. used QCT in addition to DXA in a study of 58 HIV-infected Thai children and adolescents (age 5-19 years) and age-matched healthy controls [25]. Areal BMD measured by DXA correlated only moderately well with volumetric BMD by QCT $(r=0.302)$. Although HIV-infected children and adolescents had lower areal BMD and areal BMC by DXA compared to uninfected controls, no difference in volumetric BMD by CT was observed between groups, underscoring the importance of bone size [25].

\section{Other measurement methods}

Two recent studies used peripheral QCT to assess cortical and trabecular thicknesses and volumetric BMD as well as indices of bone strength $[31,45]$. In a study by Macdonald et al., no differences in total and cortical bone area Z-scores at the tibia were observed between 31 HIV-infected subjects ages 9-18 years and a database of healthy subjects; however, ethnic-specific norms were not available [45]. Interestingly, cortical volumetric BMD appeared to be higher in those with HIV and was positively associated with non-nucleoside reverse transcriptase inhibitor (NNRTI) use. In addition, cortical thickness was negatively associated with PI use. In contrast, Yin et al. found that total and trabecular volumetricBMD and cortical and trabecular thicknesses at both the radius and tibia were between 6 and 19\% lower in young men ages 20-25 years with HIV acquired perinatally or during adolescence than healthy age-matched controls using high resolution peripheral QCT. The authors point out that these differences are similar to the magnitude observed among postmenopausal women with fractures compared to those without fractures [56]. This study also used high resolution peripheral QCT to evaluate the orientation and characteristics of plate and rod trabecular elements and revealed that HIV-infected men had significant deficiencies in plate-related parameters and 14-17\% lower bone stiffness, an accepted measure of bone strength $[57,58]$.

QUS, which estimates bone density based on the characteristics of ultrasound wave transmission through bone, has also been used in several published studies of HIV-infected children, adolescents, and young adults $[36,39,41]$. QUS devices are portable, simple to operate and do not involve exposure to radiation and may prove to be an attractive alternative for research in low-resource countries where DXA may not be available. QUS devices can evaluate the cortex of long bones at the tibia, radius or phalanges, which are largely comprised of cortical or compact bone or the calcaneus which is mainly trabecular or cancellous in nature. In a study that included children 3-17 years of age the mean phalangeal SOS $(\mathrm{m} / \mathrm{s})$, the travel time of sound waves through the region of interest, adjusted for age, skeletal age, height, and BMI was significantly lower in those with HIV infection compared to healthy controls [39]. BUA $(\mathrm{dB} / \mathrm{MHz})$, the differential attenuation of sound waves transmitted through bone, was reported to be lower in HIV-infected children with severe disease, but not those with mild-moderate disease compared to healthy controls [36]. Although the physical properties measured by QUS and DXA are not identical, they appear to be well correlated in children with HIV [41]. Mora et al. observed that radial SOS was significantly correlated with LS and WB BMC and BMD ( $r=0.57-0.60)$ after corrections for sex, weight, and height, and that tibial SOS related to all DXA BMC and BMD measurements $(\mathrm{r}=0.58-0.66)$. Z-scores by QUS (tibial and 
radial) and DXA did not differ for WB BMC and WB BMD but LS $\mathrm{BMC}$ and BMD Z-scores were significantly lower than those obtained by radial and tibial QUS as might be expected given the greater proportion of cortical bone in WB compared to the spine [41]. In addition, DXA and QUS yielded comparable results for identification of those with $<-2.0 \mathrm{Z}$-score.

\section{Risk Factors}

Several factors traditionally associated with low bone mass were identified in published studies involving HIV-infected children and adolescents, including low weight and height for age, and delayed biologic maturation [24,30,47]. We identified two studies that evaluated the relationships between lean body mass and bone [24,45]. MacDonald et al. reported that lean mass Z-score was lower in subjects with HIV compared to the reference group and was significantly associated with WB BMC as well as FN BMC and peripheral femur BMC but not LS BMC [45]. Of interest, despite lower muscle crosssectional area, a proxy of muscle force, total and cortical bone area Zscores were not significantly different from healthy controls, suggesting an intact relationship between muscle force and bone mass. Jacobson et al. also reported a positive correlation between arm muscle circumference and WB BMD; however this finding was not sustained in multivariable analysis [24]. In addition, there is evidence that lipodystrophy, which refers to a number of abnormalities in regional fat distribution that occurs in children as well as adults with HIV, is associated with lower BMC and BMD [33].

In a study conducted in the United States in which calcium amount was $20-50 \%$ below the recommended intake, O'Brien et al. reported that inadequate dietary intake of calcium contributed to reductions in bone mass [21]. This is in contrast to a recent Brazilian study in which calcium intake did not significantly influence bone mass [48]. Inadequate intake of vitamin $\mathrm{D}$ and vitamin $\mathrm{D}$ deficiency (i.e. low plasma concentrations of 25-hydroxyvitamin D) are also reported to be highly prevalent but neither has been established as a significant risk factor for low bone mass [24,47]. Further, a 2-year randomized placebo controlled trial of vitamin $\mathrm{D}$ and calcium supplementation failed to detect improvements in bone mass despite achieving adequacy in plasma concentrations of 25-hydroxyvitamin D among those receiving supplementation $[28,59]$.

Assessments of physical activity, an important determinant of muscle mass on bone accrual, have not been extensively conducted. Though DiMeglio et al. reported greater (self-reported on questionnaire) physical activity to be associated with higher LS BMD, Lima et al. did not find bone indices to be significantly associated with accelerometer-assessed levels of physical activity [30,48]. Mulligan et al. also found no statistically significant association between low bone mass and regular exercise measured by questionnaire [7]. A longitudinal study by Mora et al. found that in HIV-infected children, annual incremental changes in leg BMD were comparable to healthy children, whereas arm BMD, was reduced, suggesting a potential for weight-bearing physical activity to be important for bone development in children with HIV [35].

The question of whether HIV per se negatively affects bone mass during childhood was addressed by 4 studies. Three relatively small studies that evaluated bone mass accrual among treatment-naïve perinatally HIV-infected children and adolescents report no deficits in WB or LS BMD compared to healthy controls $[25,33,38]$. Similarly, no short-term differences in bone measures were detected among treatment-naïve and demographically similar seronegative subjects and 199 relatively recently horizontally infected males ages $14-25$ years (mean time since diagnosis 1.3 years) [7].

Among those living with HIV, associations between bone measures and plasma HIV RNA concentration (negative), CD4 count (positive), and stage of HIV (lower with advanced disease) are reported across many studies $[24,30,47,51]$. However, study results vary on some of these factors; several studies found no association between advanced stage of HIV and bone measures $[21,35,48]$ while others found a negative association [24,36]. In a study of HIV-infected children treated at a single hospital in The Netherlands with repeated DXA scans, those with higher CD4\% at the time of the scan had higher LS BMD Z-scores and those with a higher plasma HIV RNA concentration had lower LS BMD Z-scores [46]. BMD was also positively correlated with CD4 count in a recent study conducted by MacDonald et al. [45].

Exposure to antiretroviral medications was also evaluated in included studies. Two identified in our search $[7,26]$ found decreases in BMD by $2-6 \%$ following initiation of treatment-naïve patients on ART regimens [7] as well as treatment-experienced patients switching to new drug regimens due to poorly controlled HIV [26]. In a study of recently horizontally infected young men on treatment for $<6$ months, Mulligan et al. reported both lower WB BMD and Z-score WB BMC. Those on both NNRTI and protease inhibitor-containing combination ART regimens had lower WB BMC Z-scores than HIV-uninfected controls [7]. Only those on protease inhibitor-containing ART had lower BMD (hip, FN, trochanter, and Ward's) than the ART naïve group (e.g. those on NNRTI therapy did not differ from naïve) [7]. Protease inhibitors, especially older formulations, are the most extensively evaluated.in published studies. Some, but not all studies in which categorical analyses of exposure to any drug in the protease inhibitor class, reported associations with decreased bone acquisition $[7,21,24,30,35,44]$. A report from Bunders et al., however, indicates that different protease inhibitor agents may affect bone development differently; for example, nelfinavir was positively associated with bone mass indices while ritonavir-boosted lopinavir was negatively associated [46]. An association between use of ritonavir or ritonavirboosted protease inhibitors and lower measures of bone mass has also been reported $[6,30,44,48]$. Studies involving subjects on established ART, reported lower BMC in children related to exposure to drugs in the nucleoside reverse transcriptase inhibitor class including stavudine [44,46] and zidovudine [6] as well as higher BMD associated with NNRTI drug class such as nevirapine [6,24].

Some, but not all studies indicate that initiation of tenofovir adversely affects bone acquisition in HIV-infected youth switching to tenofovir among those on established ART may also affect bones negatively, although there may be less impact among those who are virologically controlled at the time of switch compared to those who are switched under less optimal conditions. Schtscherbyna et al. reported lower LS and WB BMD Z-scores among those on TDF, and the length of time of TDF was indirectly correlated with LS and WB BMD Z-score [49]. A non-comparative study reported a decline in BMD of $>6 \%$ at the FN, TH, and LS, during the initial 24-48 weeks in 6 of 15 treatment experienced children ages 12-16 years who were switched to tenofovir [26]. However, in a 48 week randomized trial involving 90 adolescents ages 12-18 years on failing ART regimens who were switched to optimized background regimen with and without tenofovir demonstrated a decline in LS and WB BMD in both groups during the first 24 weeks after randomization but no group difference in mean spine or WB BMD Z-score at 48 weeks. In 
addition, $18 \%$ of children switched to a tenofovir-containing regimen experienced a significant decline in LS BMD $(>4 \%)$ as compared to only $3 \%$ switched to regimens without tenofovir; however, these differences were not statistically significant [50]. Recent data also suggest that there are adverse effects on bone mass and early growth in HIV-uninfected infants with fetal exposure to tenofovir used as either part of maternal combination ART or as prophylaxis against mother to child transmission, providing additional evidence of a possible toxic effect of tenofovir to bone development $[60,61]$.

The duration of antiretroviral exposure is also significantly associated with bone mass measures in children and adolescents but not all studies agree on the direction. In a longitudinal study in the Netherlands, duration of ART was associated with an increase in LS BMD Z-score (coefficient, 0.08; $\mathrm{P}<0.001$ ); this association remained after adjusting for BMI, CD4\% and plasma HIV RNA concentration [46]. DiMeglio et al. found that LS BMD Z-scores was associated with more years on HAART $(-0.03$ per year, $\mathrm{p}=0.14)$ [30].

No histomorphometric studies have been conducted in HIVinfected children or adolescents; therefore, evaluations of pathogenic mechanisms in the studies in this review have relied on correlations with biochemical markers. Several studies found elevated concentrations of bone turnover markers (e.g. bone formation and resorption) among HIV-infected children and adolescents $[33,34,43,62]$, and were interpreted to be indicative of increased bone remodeling. Mora et al. found that HIV-infected children on ART had higher concentrations of bone alkaline phosphatase (BALP), a bone formation marker, and N-telopeptide (NTX), a bone resorption marker, than untreated HIV-infected children and HIV-uninfected children [33]. In contrast, Tan et al. reported higher bone formation, as measured by osteocalcin, in HIV-infected children than controls and lower resorption measured by carboxyterminal telopeptide of type I collagen (ICTP) in HIV-infected children on protease inhibitors (PIs) [62].

Stagi et al. reported that reduced bone mass and quality in HIVinfected children were related to reduced IGF-1, a potent modulator of osteoblast-osteoclast interactions [63], longitudinal bone growth and bone mass acquisition during childhood [64,65], and possibly related to overproduction of IL-6 [36], similar to findings from an earlier, smaller study [34]. Finally, an increased incidence of osteonecrosis of the hip (i.e. Legg-Calvé-Perthes disease) is reported in one study [32]. A single study evaluating the risk of fracture in HIV-infected children did not detect an increase in fracture rate [29]. This study compared rates of fracture in a cohort of $1326 \mathrm{HIV}$-infected and $649 \mathrm{HIV}$ uninfected children with a mean age of 5.8-7.1 with a median of 2.26-4.97 years and found similar rates of fracture in the HIV-infected and uninfected groups (1.2 vs. 1.1 per 1000 person-years) [29].

\section{Discussion}

We systematically reviewed the literature to identify studies that reported on bone imaging or bone fractures in HIV-infected children, adolescents, or young adults. Although the studies vary greatly with respect to comparison sources, adjustment techniques for body size or growth retardation, and highlighted risk factors, most studies we reviewed found that measures of bone mass are reduced in children and adolescents with HIV. Reductions in bone mass are reported in association with antiretroviral medications, with specific antiretroviral classes and agents such as protease inhibitors and tenofovir appearing more prominently in the literature than other classes or agents, but study results are inconsistent.

The heterogeneity with respect to the sources used for comparison in the published studies poses a problem for assessing the severity of deficits in bone mass acquisition and the extent to which the observed reductions in bone mass may be attributable to factors other than HIV known to be important to bone accrual [66], such as small size for age and delayed biologic maturation which frequently accompany HIV infection in children and adolescents. It also limits comparison of findings across studies. There does not appear to be a single optimal comparison group and the best choice may vary with the questions being asked. For example, while inclusion of siblings of HIV-infected children as controls may be optimal for race/ethnicity, genetics, and other factors, this approach may not provide the best estimation of the status of bone mass as compared to the overall population. For example, Jacobson et al. found that children with HIV had lower WB BMD than expected compared to a normative database but no difference when compared to siblings [24]. Another potential problem with this approach is that some uninfected siblings may have had intrauterine exposure to antiretrovirals and the inflammatory environment of maternal HIV infection. Use of large longitudinal normative databases that have recently become available [67-69] as well as inclusion of additional healthy control subjects recruited for study purposes can overcome these limitations.

The studies included in this review also differed in the approaches used for adjusting for bone size, height, lean body mass, skeletal age, and state of biologic maturation. DXA does not measure the anteroposterior diameter of bone and provides only a twodimensional areal estimate of $\mathrm{BMD}\left(\mathrm{g} / \mathrm{cm}^{2}\right)$, which may underestimate true volumetric BMD $\left(\mathrm{g} / \mathrm{cm}^{3}\right)$ particularly in children with impaired growth or maturational delays [70]. The International Society for Clinical Densitometry advises that in children with short stature, whole body BMC and areal BMD results should be adjusted using height-for-age Z-score (HAZ), and either BMAD or HAZ used for LS results [71]. The literature we reviewed used a number of different approaches to account for smaller skeletal size and maturational delays. Age, sex, height, weight, BMI, race, and pubertal stage were variably included and several studies adjusted for bone age $[25,38]$. Only Jacobson et al. included HAZ which in healthy children is the strategy least likely to result in biased estimates of BMC and BMD in older, shorter children (i.e. $\mathrm{HAZ}<-1.0$ ) [66].

Although in vitro studies suggest that HIV-specific proteins alter osteoblast function by means of disrupting the differentiation of precursor stem cells into osteoblasts, stimulating osteoblast apoptosis and altering osteoblast activity, evidence from clinical studies to support a direct role for HIV is limited, and distinguishing direct effects of HIV from those due to inflammation poses a challenge. HIV infection is characterized by chronic immune activation with polyclonal B cell activation, production of cytokines and increased $\mathrm{T}$ cell activation and turnover [72,73].

Chronic immune activation is among the putative causes of bone loss in adults with HIV but has not been carefully studied in children and adolescents. Despite effective ART and viral suppression, the proportion of activated (CD38+) T cells and serum pro-inflammatory cytokines remains elevated [74-78]. Chronic immune activation and increased levels of pro-resorptive cytokines like TNFa, IL-6 and RANKL are thought to increase osteoclastic resorption. In addition, some antiretroviral agents may also reduce osteoblast activity via induction of inflammatory activity [9]. If osteoblast-mediated bone 
formation is unable to match or exceed resorption during periods of growth, then inadequate bone acquisition could result. The role of persistent residual immune activation despite viral suppression on bone formation and resorption during growth is an additional important line of investigation.

Some of the inconsistencies regarding ART exposures and bone measures in the reviewed studies may be attributed to variability in time elapsed between ART initiation or change in ART regimen and the ascertainment of bone measurements. Evidence from studies conducted in adults [79-83], as well as studies identified in our search $[7,26]$, indicate that BMD declines during initial phases of treatment in relation to a number of different ART regimens. In addition, as antiretroviral drugs are typically used in combination and often sequentially, attributing a specific bone outcome finding to a single drug is difficult in an observational study. Further, many of these observational studies have low power to detect differences in bone accrual by treatment regimen due to the inclusion of small numbers of subjects on a multiplicity of drug combinations [33,44]. In some studies, specific drug agents were assessed separately while in others analyses were conducted combining multiple members from a pharmacologic class, which may contribute to inconsistent results in the literature.

Several antiretroviral agents may be of particular clinical and public health importance due to their potential impact on bone metabolism and their widespread use and warrant further study. Ritonavir-boosted protease inhibitors, among the more widely used medications for children and adolescents, is associated with declines in BMD. In addition, a meta-analysis of adult studies that revealed an association between protease inhibitor use and low BMD and in vitro studies suggesting that ritonavir alters osteoclast as well as osteoblast gene expression resulting in dysregulation of cell differentiation, expression of growth factors, and enzymes that favour bone resorption $[8,9,84]$ provides additional rationale to investigate further. Questions also remain regarding short- and long-term effects of tenofovir, another important drug agent increasingly used in children and adolescents. There are several potential mechanisms for adverse bone effects for tenofovir. In vitro studies suggest tenofovir may have a direct effect on bone formation by affecting osteoblast proliferation and increasing apoptosis [85], and clinical studies suggest that tenofovir induces functional vitamin $\mathrm{D}$ deficiency [86].

Although disturbances in body composition in paediatric HIV are well described, the relationships between fat or lean tissue compartments and bone mass have not been evaluated extensively $[87,88]$. In light of the potential importance of increased muscle forces and mechanical loading on bone development [89], assessments of the role of physical activity warrant further examination as this may have therapeutic implications.

As presented in Figure 2, osteoporosis and fracture later in life may be increased by impaired bone acquisition early in life. The accrual of bone mass takes place from foetal life all the way through early adulthood, and the majority (95\%) of PBM is attained by young adulthood. One recent study suggests that those who acquire HIV early in life do not achieve optimal PBM [31]. PBM is a key predictor of osteoporosis and fracture risk [15]. Mathematical models indicate that relatively small increases (10\%) in PBM acquisition in healthy females could delay onset of osteoporosis by as many as 13 years [90]. The reductions in PBM and micro-architectural abnormalities observed by Yin et al. may have important implications for increasing future risk of fracture and requires further study.
There is a substantial gap in studies from low- and middle-income countries, where the majority of children with HIV live, but where access to bone imaging by DXA is limited. Alternative methods such as QUS could facilitate expansion of bone-related research in these settings. There is also a paucity of evaluation of interventions for optimizing bone accrual among HIV-infected children and adolescents either by selection of "bone sparing" ART, maximizing weight bearing physical activity. Although results of one study of vitamin $\mathrm{D}$ and calcium supplementation in children and adolescents did not find an effect [59], vitamin D supplementation may prove useful as a specific means to prevent bone loss with tenofovir exposure [86].

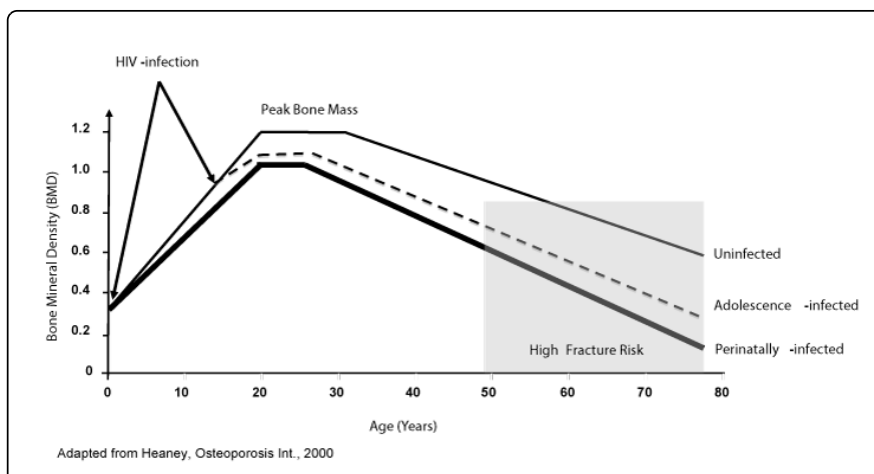

Figure 2: Hypothetical evolution of bone acquisition with HIV infection early in life.

There is a strong need for further, high-quality research, particularly in resource-constrained settings. Studies to determine fracture risk as well as to mitigate the adverse effects on bone of HIV infection, co-morbid conditions and associated therapeutics are important for the over 8 million children and youth currently living with HIV worldwide.

\section{References}

1. Triant VA, Brown TT, Lee H, Grinspoon SK (2008) Fracture prevalence among human immunodeficiency virus (HIV)-infected versus non-HIVinfected patients in a large U.S. healthcare system. J Clin Endocrinol Metab 93: 3499-3504.

2. Womack JA, Goulet JL, Gibert C, Brandt C, Chang CC, et al. (2011) Increased risk of fragility fractures among HIV infected compared to uninfected male veterans. PLoS One 6: e17217.

3. Hasse B, Ledergerber B, Furrer H, Battegay M, Hirschel B, et al. (2011) Morbidity and aging in HIV-infected persons: the Swiss HIV cohort study. Clin Infect Dis 53: 1130-1139.

4. Young B, Dao CN, Buchacz K, Baker R, Brooks JT; HIV Outpatient Study (HOPS) Investigators (2011) Increased rates of bone fracture among HIV-infected persons in the HIV Outpatient Study (HOPS) compared with the US general population, 2000-2006. Clin Infect Dis 52: 1061-1068.

5. Shiau S, Broun EC, Arpadi SM, Yin MT (2013) Incident fractures in HIV-infected individuals: a systematic review and meta-analysis. AIDS 27: 1949-1957.

6. Jacobson DL, Lindsey JC, Gordon CM, Moye J, Hardin DS, et al. (2010) Total body and spinal bone mineral density across Tanner stage in perinatally HIV-infected and uninfected children and youth in PACTG 1045. AIDS 24: 687-696.

7. Mulligan K, Harris DR, Emmanuel P, Fielding RA, Worrell C, et al. (2012) Low bone mass in behaviorally HIV-infected young men on 
antiretroviral therapy: Adolescent Trials Network Study 021B. Clin Infect Dis 55: 461-468

8. Fakruddin JM, Laurence J (2005) HIV-1 Vpr enhances production of receptor of activated NF-kappaB ligand (RANKL) via potentiation of glucocorticoid receptor activity. Arch Virol 150: 67-78

9. Malizia AP, Cotter E, Chew N, Powderly WG, Doran PP (2007) HIV protease inhibitors selectively induce gene expression alterations associated with reduced calcium deposition in primary human osteoblasts. AIDS Res Hum Retroviruses 23: 243-250

10. Mondy K, Yarasheski K, Powderly WG, Whyte M, Claxton S, et al. (2003) Longitudinal evolution of bone mineral density and bone markers in human immunodeficiency virus-infected individuals. Clin Infect Dis 36: 482-490.

11. Wang MW, Wei S, Faccio R, Takeshita S, Tebas P, et al. (2004) The HIV protease inhibitor ritonavir blocks osteoclastogenesis and function by impairing RANKL-induced signaling. J Clin Invest 114: 206-213.

12. Wong BR, Besser D, Kim N, Arron JR, Vologodskaia M, et al. (1999) TRANCE, a TNF family member, activates Akt/PKB through a signaling complex involving TRAF6 and c-Src. Mol Cell 4: 1041-1049.

13. Cotter EJ, Chew N, Powderly WG, Doran PP (2011) HIV type 1 alters mesenchymal stem cell differentiation potential and cell phenotype ex vivo. AIDS Res Hum Retroviruses 27: 187-199.

14. Walker Harris V, Brown TT (2012) Bone loss in the HIV-infected patient: evidence, clinical implications, and treatment strategies. J Infect Dis 205 Suppl 3: S391-398.

15. Heaney RP, Abrams S, Dawson-Hughes B, Looker A, Marcus R, et al. (2000) Peak bone mass. Osteoporos Int 11: 985-1009.

16. Bailey DA, Martin AD, McKay HA, Whiting S, Mirwald R (2000) Calcium accretion in girls and boys during puberty: a longitudinal analysis. J Bone Miner Res 15: 2245-2250.

17. Theintz G, Buchs B, Rizzoli R, Slosman D, Clavien H, et al. (1992) Longitudinal monitoring of bone mass accumulation in healthy adolescents: evidence for a marked reduction after 16 years of age at the levels of lumbar spine and femoral neck in female subjects. J Clin Endocrinol Metab 75: 1060-1065.

18. Bhaskaran K, Hamouda O, Sannes M, Boufassa F, Johnson AM, et al. (2008) Changes in the risk of death after HIV seroconversion compared with mortality in the general population. JAMA 300: 51-59.

19. Lohse N, Hansen AB, Pedersen G, Kronborg G, Gerstoft J, et al. (2007) Survival of persons with and without HIV infection in Denmark, 1995-2005. Ann Intern Med 146: 87-95.

20. UNAIDS (2013) Global report: UNAIDS report on the global AIDS epidemic.

21. O'Brien KO, Razavi M, Henderson RA, Caballero B, Ellis KJ (2001) Bone mineral content in girls perinatally infected with HIV. Am J Clin Nutr 73: 821-826.

22. Arpadi SM, Horlick M, Thornton J, Cuff PA, Wang J, et al. (2002) Bone mineral content is lower in prepubertal HIV-infected children. J Acquir Immune Defic Syndr 29: 450-454.

23. Hazra R, Gafni RI, Maldarelli F, Balis FM, Tullio AN et al. (2005) Tenofovir disoproxil fumarate and an optimized background regimen of antiretroviral agents as salvage therapy for pediatric HIV infection. Pediatrics 116: e846-854

24. Jacobson DL, Spiegelman D, Duggan C, Weinberg GA, Bechard L, et al. (2005) Predictors of bone mineral density in human immunodeficiency virus-1 infected children. J Pediatr Gastroenterol Nutr 41: 339-346.

25. Pitukcheewanont P, Safani D, Church J, Gilsanz V (2005) Bone measures in HIV-1 infected children and adolescents: disparity between quantitative computed tomography and dual-energy X-ray absorptiometry measurements. Osteoporos Int 16: 1393-1396

26. Gafni RI, Hazra R, Reynolds JC, Maldarelli F, Tullio AN et al. (2006) Tenofovir disoproxil fumarate and an optimized background regimen of antiretroviral agents as salvage therapy: impact on bone mineral density in HIV-infected children. Pediatrics 118: e711-718
27. Purdy JB, Gafni RI, Reynolds JC, Zeichner S, Hazra R (2008) Decreased bone mineral density with off-label use of tenofovir in children and adolescents infected with human immunodeficiency virus. J Pediatr 152: 582-584.

28. Arpadi SM, McMahon DJ, Abrams EJ, Bamji M, Purswani M, et al. (2012) Effect of supplementation with cholecalciferol and calcium on 2-y bone mass accrual in HIV-infected children and adolescents: a randomized clinical trial. Am J Clin Nutr 95: 678-685.

29. Siberry GK, Li H, Jacobson D; Pediatric AIDS Clinical Trials Group (PACTG) 219/219C Study (2012) Fracture risk by HIV infection status in perinatally HIV-exposed children. AIDS Res Hum Retroviruses 28: 247-250.

30. DiMeglio LA, Wang J, Siberry GK, Miller TL, Geffner ME, et al. (2013) Bone mineral density in children and adolescents with perinatal HIV infection. AIDS 27: 211-220.

31. Yin MT, Lund E, Shah J, Zhang CA, Foca M, et al. (2014) Lower peak bone mass and abnormal trabecular and cortical microarchitecture in young men infected with HIV early in life. AIDS 28: 345-353.

32. Gaughan DM, Mofenson LM, Hughes MD, Seage GR 3rd, Ciupak GL, et al. (2002) Osteonecrosis of the hip (Legg-Calvé-Perthes disease) in human immunodeficiency virus-infected children. Pediatrics 109: E74-74.

33. Mora S, Sala N, Bricalli D, Zuin G, Chiumello G, et al. (2001) Bone mineral loss through increased bone turnover in HIV-infected children treated with highly active antiretroviral therapy. AIDS 15: 1823-1829.

34. Zamboni G, Antoniazzi F, Bertoldo F, Lauriola S, Antozzi L, et al. (2003) Altered bone metabolism in children infected with human immunodeficiency virus. Acta Paediatr 92: 12-16.

35. Mora S, Zamproni I, Beccio S, Bianchi R, Giacomet V et al. (2004) Longitudinal changes of bone mineral density and metabolism in antiretroviral-treated human immunodeficiency virus-infected children. J Clin Endocrinol Metab 89: 24-28

36. Stagi S, Bindi G, Galluzzi F, Galli L, Salti R, et al. (2004) Changed bone status in human immunodeficiency virus type 1 (HIV-1) perinatally infected children is related to low serum free IGF-I. Clin Endocrinol (Oxf) 61: 692-699.

37. Giacomet V, Mora S, Martelli L, Merlo M, Sciannamblo M, et al. (2005) A 12-month treatment with tenofovir does not impair bone mineral accrual in HIV-infected children. J Acquir Immune Defic Syndr 40: 448-450.

38. Mora S, Zamproni I, Giacomet V, Cafarelli L, Figini C, et al. (2005) Analysis of bone mineral content in horizontally HIV-infected children naïve to antiretroviral treatment. Calcif Tissue Int 76: 336-340.

39. Rosso R, Vignolo M, Parodi A, Di Biagio A, Sormani MP, et al. (2005) Bone quality in perinatally HIV-infected children: role of age, sex, growth, HIV infection, and antiretroviral therapy. AIDS Res Hum Retroviruses 21: 927-932.

40. Mora S, Zamproni I, Cafarelli L, Giacomet V, Erba P, et al. (2007) Alterations in circulating osteoimmune factors may be responsible for high bone resorption rate in HIV-infected children and adolescents. AIDS 21: 1129-1135.

41. Mora S, Viganò A, Cafarelli L, Pattarino G, Giacomet V, et al. (2009) Applicability of quantitative ultrasonography of the radius and tibia in HIV-infected children and adolescents. J Acquir Immune Defic Syndr 51: 588-592.

42. Rosso R, Parodi A, Torrisi C, De Terlizzi F, Viscoli C, et al. (2010) A tailored dose of tenofovir could reduce its impact on bone mass in HIV Type 1-infected children and adolescents: a report from 5 years of clinical experience. AIDS Res Hum Retroviruses 26: 1265-1266.

43. Viganò A, Zuccotti GV, Puzzovio M, Pivetti V, Zamproni I, et al. (2010) Tenofovir disoproxil fumarate and bone mineral density: a 60-month longitudinal study in a cohort of HIV-infected youths. Antivir Ther 15: 1053-1058.

44. Zuccotti G, Viganò A, Gabiano C, Giacomet V, Mignone F, et al. (2010) Antiretroviral therapy and bone mineral measurements in HIV-infected youths. Bone 46: 1633-1638. 
45. Macdonald HM, Chu J, Nettlefold L, Maan EJ, Forbes JC, et al. (2013) Bone geometry and strength are adapted to muscle force in children and adolescents perinatally infected with HIV. J Musculoskelet Neuronal Interact 13: 53-65.

46. Bunders MJ, Frinking O, Scherpbier HJ, van Arnhem LA, van Eck-Smit BL, et al. (2013) Bone mineral density increases in HIV-infected children treated with long-term combination antiretroviral therapy. Clin Infect Dis 56: 583-586.

47. Puthanakit $T$, Saksawad $R$, Bunupuradah $T$, Wittawatmongkol $O$, Chuanjaroen T, et al. (2012) Prevalence and risk factors of low bone mineral density among perinatally HIV-infected Thai adolescents receiving antiretroviral therapy. J Acquir Immune Defic Syndr 61: 477-483.

48. Lima LR, Silva RC, Giuliano Ide C, Sakuno T, Brincas SM, et al. (2013) Bone mass in children and adolescents infected with human immunodeficiency virus. J Pediatr (Rio J) 89: 91-99.

49. Schtscherbyna A, Pinheiro MF, Mendonça LM, Gouveia C, Luiz RR, et al (2012) Factors associated with low bone mineral density in a Brazilian cohort of vertically HIV-infected adolescents. Int J Infect Dis 16 e872-878.

50. Della Negra M, de Carvalho AP, de Aquino MZ, da Silva MT, Pinto J, et al. (2012) A randomized study of tenofovir disoproxil fumarate in treatment-experienced HIV-1 infected adolescents. Pediatr Infect Dis J 31: 469-473.

51. Negredo E, Domingo P, Ferrer E, Estrada V, Curran A, et al. (2014) Peak bone mass in young HIV-infected patients compared with healthy controls. J Acquir Immune Defic Syndr 65: 207-212.

52. Faulkner KG, Roberts LA, McClung MR (1996) Discrepancies in normative data between Lunar and Hologic DXA systems. Osteoporos Int 6: 432-436.

53. Writing Group for the ISCD Position Development Conference (2004) Diagnosis of osteoporosis in men, premenopausal women, and children. J Clin Densitom 7: 17-26.

54. Carter DR, Bouxsein ML, Marcus R (1992) New approaches for interpreting projected bone densitometry data. J Bone Miner Res 7: 137-145.

55. Katzman DK, Bachrach LK, Carter DR, Marcus R (1991) Clinical and anthropometric correlates of bone mineral acquisition in healthy adolescent girls. J Clin Endocrinol Metab 73: 1332-1339.

56. Sornay-Rendu E, Boutroy S, Munoz F, Delmas PD (2007) Alterations of cortical and trabecular architecture are associated with fractures in postmenopausal women, partially independent of decreased BMD measured by DXA: the OFELY study. J Bone Miner Res 22: 425-433.

57. Liu XS, Sajda P, Saha PK, Wehrli FW, Guo XE (2006) Quantification of the roles of trabecular microarchitecture and trabecular type in determining the elastic modulus of human trabecular bone. J Bone Miner Res 21: 1608-1617.

58. Liu XS, Shane E, McMahon DJ, Guo XE (2011) Individual trabecula segmentation (ITS)-based morphological analysis of microscale images of human tibial trabecular bone at limited spatial resolution. J Bone Miner Res 26: 2184-2193

59. Arpadi SM, McMahon D, Abrams EJ, Bamji M, Purswani M, et al. (2009) Effect of bimonthly supplementation with oral cholecalciferol on serum 25-hydroxyvitamin D concentrations in HIV-infected children and adolescents. Pediatrics 123: e121-126.

60. Nurutdinova D, Onen NF, Hayes E, Mondy K, Overton ET (2008) Adverse effects of tenofovir use in HIV-infected pregnant women and their infants. Ann Pharmacother 42: 1581-1585.

61. Siberry GK, Jacobson DL, Kalkwarf HJ (2014) Lower Newborn Bone Mineral Content Associated With Maternal? Use of Tenofovir Disoproxil Fumarate. Conference on Retroviruses and Opportunistic Infections (CROI), Boston, MA, USA

62. Tan BM, Nelson RP Jr., James-Yarish M, Emmanuel PJ, Schurman SJ (2001) Bone metabolism in children with human immunodeficiency virus infection receiving highly active anti-retroviral therapy including a protease inhibitor. J Pediatr 139:447-451
63. Le Roith D, Bondy C, Yakar S, Liu JL, Butler A (2001) The somatomedin hypothesis: 2001. Endocr Rev 22: 53-74.

64. Yanovski JA, Sovik KN, Nguyen TT, Sebring NG (2000) Insulin-like growth factors and bone mineral density in African American and White girls. J Pediatr 137: 826-832.

65. Zofková I (2003) Pathophysiological and clinical importance of insulinlike growth factor-I with respect to bone metabolism. Physiol Res 52: 657-679.

66. Zemel BS, Leonard MB, Kelly A, Lappe JM, Gilsanz V, et al. (2010) Height adjustment in assessing dual energy $\mathrm{x}$-ray absorptiometry measurements of bone mass and density in children. J Clin Endocrinol Metab 95: 1265-1273.

67. Kalkwarf HJ, Gilsanz V, Lappe JM, Oberfield S, Shepherd JA, et al. (2010) Tracking of bone mass and density during childhood and adolescence. J Clin Endocrinol Metab 95: 1690-1698.

68. Kalkwarf HJ, Zemel BS, Gilsanz V, Lappe JM, Horlick M, et al. (2007) The bone mineral density in childhood study: bone mineral content and density according to age, sex, and race. J Clin Endocrinol Metab 92: 2087-2099.

69. Kelly TL, Wilson KE, Heymsfield SB (2009) Dual energy X-Ray absorptiometry body composition reference values from NHANES. PLoS One 4: e7038.

70. Majaliwa ES, Mohn A, Chiarelli F (2009) Growth and puberty in children with HIV infection. J Endocrinol Invest 32: 85-90.

71. Crabtree NJ, Arabi A, Bachrach LK, Fewtrell M, El-Hajj Fuleihan G, et al. (2014) Dual-energy X-ray absorptiometry interpretation and reporting in children and adolescents: the revised 2013 ISCD Pediatric Official Positions. J Clin Densitom 17: 225-242.

72. Brenchley JM, Price DA, Schacker TW, Asher TE, Silvestri G, et al. (2006) Microbial translocation is a cause of systemic immune activation in chronic HIV infection. Nat Med 12: 1365-1371.

73. Douek DC, Picker LJ, Koup RA (2003) T cell dynamics in HIV-1 infection. Annu Rev Immunol 21: 265-304.

74. Sandler NG, Wand H, Roque A, Law M, Nason MC, et al. (2011) Plasma levels of soluble CD14 independently predict mortality in HIV infection. J Infect Dis 203: 780-790.

75. Cassol E, Malfeld S, Mahasha P, van der Merwe S, Cassol S et al. (2010) Persistent microbial translocation and immune activation in HIV-1infected South Africans receiving combination antiretroviral therapy. J Infect Dis 202: 723-733

76. Lichtfuss GF, Cheng WJ, Farsakoglu Y, Paukovics G, Rajasuriar R, et al. (2012) Virologically suppressed HIV patients show activation of NK cells and persistent innate immune activation. J Immunol 189: 1491-1499.

77. Lichtfuss GF, Hoy J, Rajasuriar R, Kramski M, Crowe SM, et al. (2011) Biomarkers of immune dysfunction following combination antiretroviral therapy for HIV infection. Biomark Med 5: 171-186.

78. Lederman MM, Funderburg NT, Sekaly RP, Klatt NR, Hunt PW (2013) Residual immune dysregulation syndrome in treated HIV infection. Adv Immunol 119: 51-83.

79. Gallant JE, Staszewski S, Pozniak AL, DeJesus E, Suleiman JM, et al. (2004) Efficacy and safety of tenofovir DF vs stavudine in combination therapy in antiretroviral-naive patients: a 3-year randomized trial. JAMA 292: 191-201.

80. Brown TT, McComsey GA, King MS, Qaqish RB, Bernstein BM, et al. (2009) Loss of bone mineral density after antiretroviral therapy initiation, independent of antiretroviral regimen. J Acquir Immune Defic Syndr 51: 554-561.

81. van Vonderen MG, Lips P, van Agtmael MA, Hassink EA, Brinkman K, et al. (2009) First line zidovudine/lamivudine/lopinavir/ritonavir leads to greater bone loss compared to nevirapine/lopinavir/ritonavir. AIDS 23: 1367-1376.

82. Stellbrink HJ, Orkin C, Arribas JR, Compston J, Gerstoft J, et al. (2010) Comparison of changes in bone density and turnover with abacavirlamivudine versus tenofovir-emtricitabine in HIV-infected adults: 48week results from the ASSERT study. Clin Infect Dis 51: 963-972. 
Citation: $\quad$ Arpadi SM, Shiau S, Arpadi CM, Yin MT (2014) Bone Health in HIV-Infected Children, Adolescents and Young Adults: A Systematic Review. J AIDS Clin Res 5: 374. doi:10.4172/2155-6113.1000374

Page 14 of 14

83. McComsey GA, Kitch D, Daar ES, Tierney C, Jahed NC, et al. (2011) Bone mineral density and fractures in antiretroviral-naive person randomized to receive abacavir-lamivudine or tenofovir disoproxil fumarate-emtricitabine along with efavirenz or atazanavir-ritonavir: Aids Clinical Trials Group A5224s, a substudy of ACTG A5202. J Infect Dis 203: 1791-1801.

84. Jain RG, Lenhard JM (2002) Select HIV protease inhibitors alter bone and fat metabolism ex vivo. J Biol Chem 277: 19247-19250.

85. Grigsby IF, Pham L, Mansky LM, Gopalakrishnan R, Carlson AE, et al. (2010) Tenofovir treatment of primary osteoblasts alters gene expression profiles: implications for bone mineral density loss. Biochem Biophys Res Commun 394: 48-53.

86. Havens PL, Stephensen CB, Hazra R, Flynn PM, Wilson CM, et al. (2012) Vitamin D3 decreases parathyroid hormone in HIV-infected youth being treated with tenofovir: a randomized, placebo-controlled trial. Clin Infect Dis 54: 1013-1025.
87. Arpadi SM, Horlick MN, Wang J, Cuff P, Bamji M, et al. (1998) Body composition in prepubertal children with human immunodeficiency virus type 1 infection. Arch Pediatr Adolesc Med 152: 688-693.

88. Arpadi S, Shiau S, Strehlau R, Martens L, Patel F, et al. (2013) Metabolic abnormalities and body composition of HIV-infected children on Lopinavir or Nevirapine-based antiretroviral therapy. Arch Dis Child 98: 258-264.

89. Frost HM (2000) Muscle, bone, and the Utah paradigm: a 1999 overview. Med Sci Sports Exerc 32: 911-917.

90. Hernandez CJ, Beaupré GS, Carter DR (2003) A theoretical analysis of the relative influences of peak BMD, age-related bone loss and menopause on the development of osteoporosis. Osteoporos Int 14: 843-847. 